\title{
A efetividade do tratamento para dor utilizando auriculoterapia: um artigo de
}

\section{revisão}

The effectiveness of pain treatment using auriculotherapy: a review article

La efectividad del tratamiento del dolor con auriculoterapia: un artículo de revisión

Recebido: 12/09/2021 | Revisado: 20/09/2021 | Aceito: 22/09/2021 | Publicado: 25/09/2021

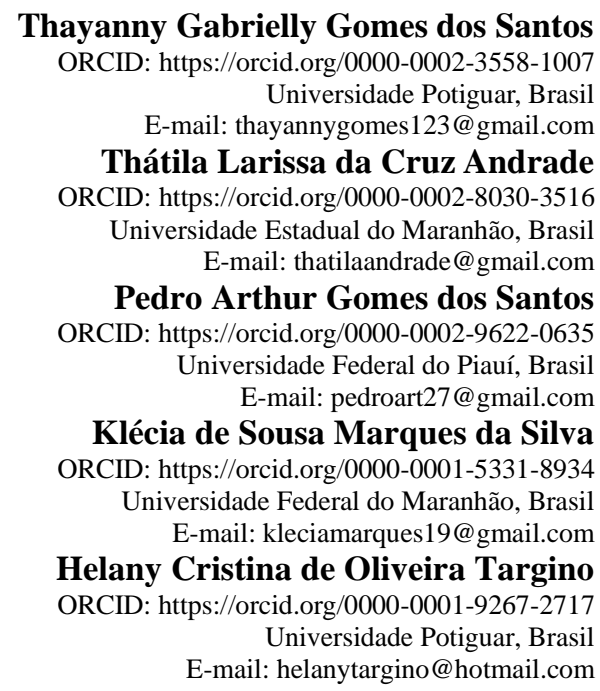

\begin{abstract}
Resumo
A auriculoterapia, microssistema da acupuntura, constitui-se como um principal método terapêutico em tratamentos de dores. O uso de sua prática dentro da medicina tradicional chinesa é datado a décadas, onde são feitos estímulos em determinadas áreas da orelha, e estes possuem reflexos no corpo. Este estudo tratou-se de uma pesquisa bibliográfica descritiva que tem por objetivo geral realizar uma revisão literária, onde possa ser identificado como auriculoterapia é utilizada no processo terapêutico de analgesia em casos de dor, e por objetivos específicos constatar a eficácia do tratamento com auriculoterapia nas algias; definir quais os pontos do pavilhão auricular são mais utilizados; e citar os meios usados para estimulação dos pontos. A coleta de dados foi realizada, baseados em 7 artigos encontrados em base de dados digitais (Google Acadêmico e SciELO), de forma sistematizada e colocados em forma de quadros. Constatou-se através da análise que houve grande significância do uso da auriculoterapia como método de analgesia.

Palavras-chave: Medicina tradicional chinesa; Acupuntura; Auriculoterapia; Dor; Analgesia.
\end{abstract}

\begin{abstract}
Auriculotherapy, an acupuncture microsystem, is a major therapeutic method in pain treatments. The use of its practice within traditional Chinese medicine dates back to decades, where stimuli are made in certain areas of the ear, and these are reflected in the body. This study was a descriptive bibliographical research that has as general objective to carry out a literature review where it can be identified as auriculotherapy is used as a therapeutic process of analgesia in cases of pain, and for specific objectives to verify the effectiveness of treatment with auriculotherapy in pain ; define which pinna points are most used; and mention the means used to stimulate the points. Data collection was performed, based on 7 articles found in a digital database (Google Academic and SciELO), in a systematic way and placed in a table. It was found through the analysis that there was great significance in the use of auriculotherapy as a method of analgesia.
\end{abstract}

Keywords: Traditional chinese medicine; Acupuncture; Auriculotherapy; Pain; Analgesia.

\section{Resumen}

La auriculoterapia, un microsistema de acupuntura, es un método terapéutico importante para el tratamiento del dolor. El uso de su práctica dentro de la medicina tradicional china se remonta a décadas, donde se realizan estímulos en determinadas zonas del oído, y estos tienen un reflejo en el cuerpo. El presente estudio fue una investigación bibliográfica descriptiva que tiene como objetivo general realizar una revisión de la literatura donde se pueda identificar como la auriculoterapia se utiliza como un proceso terapéutico de analgesia en casos de dolor, y con 
objetivos específicos verificar la efectividad del tratamiento con auriculoterapia. en dolor definir qué puntos del pabellón auricular se utilizan más; y mencione los medios utilizados para estimular los puntos. La recolección de datos se realizó, a partir de 7 artículos encontrados en una base de datos digital (Google Academic y SciELO), de manera sistemática y colocados en unos marcos. A través del análisis se encontró que el uso de la auriculoterapia como método de analgesia tiene una gran importancia.

Palabras clave: Medicina tradicional china; Acupuntura; Auriculoterapia; Dolor; Analgesia.

\section{Introdução}

A Medicina Tradicional Chinesa (MTC) é praticada há cerca de 5.000 anos no Oriente e atualmente é utilizada em vários países ocidentais, como forma de amplificar as alternativas de condutas terapêuticas. É vista como uma contribuição extremamente importante para a melhoria das condições de vida da população (Brisola et al., 2016).

Dentre as numerosas técnicas da MTC, situa-se a acupuntura, cujo termo é derivado do latim: agulha (acus) e puntura (puntura). A terapia declara que em nosso corpo existem aproximadamente 360 acupontos de meridianos e a maioria deles está associado com estruturas do sistema nervoso periférico, feixes periféricos e vasos sanguíneos. Ao se introduzir a agulha ativase os receptores dos dendritos, localizados nos neurônios sensoriais da pele, músculos e tecidos moles. Essa estimulação gera diversos sinais elétricos, na qual são conhecidos como potencial de ação, que deslocam-se até os dendritos, chegam ao corpo celular, posteriormente ao axônio e finalmente ao cérebro por meio das sinapses (Ma, Ma, Cho, 2006).

A analgesia proporcionada pela acupuntura envolve a estimulação de nervos de pequeno diâmetro e limiar diferenciado. Esses nervos enviam mensagens à medula espinhal, o que ativa neurônios do tronco cerebral (área cinzenta periaquedutal) e do hipotálamo, disparando mecanismos de opioides endógenos (Meirelles et al., 2009).

Dentre as terapias alternativas, além da acupuntura sistêmica, na qual as agulhas são inseridas em diferentes acupontos dos meridianos energéticos, em várias áreas do corpo, com efeitos locais e gerais, há também a aurículo-acupuntura e a eletro-aurículoacupuntura, técnicas nas quais as agulhas são inseridas em regiões específicas na orelha, também objetivando ativar a liberação de substâncias químicas endógenas capazes de exercerem efeito analgésico (Boleta - Ceranto et al., 2008).

Auriculoterapia é uma área da Acupuntura voltada ao tratamento das doenças físicas e mentais, onde o procedimento é realizado por meio de estímulos, cujos pontos são localizados no pavilhão auricular. Cada orelha possui pontos de reflexos que equivalem a todos os órgãos, assim como, funções do corpo. Ao se efetuar a sensibilização desses pontos por agulhas de acupuntura, o cérebro ganha um impulso que provoca uma série de fenômenos físicos pertinentes com a área do corpo, produzindo a cura (Souza, 2012).

Ademais, tal prática também é indicada para o tratamento de várias patologias: dolorosas, inflamatórias, endocrinometabólicas e do sistema urogenital, doenças de caráter funcional, crônicas, infectocontagiosas, entre outras. São indicadas em eventualidades, onde o paciente tem a necessidade de alívio imediato de dor, dores pungentes, agudas e crônicas, perturbações psíquicas como ansiedade e depressão, angústia, desconcentração, vertigens, disfemia, perturbações do sistema autônomo, intoxicações por uso de drogas, tabaco e medicações (Kurebayashi et al., 2012).

À proporção que se estimula o ponto reflexo na orelha, é possível promover uma ação de alívio de sintomas em partes distantes do corpo (Gori, \& Firenzuoli, 2007).

A dor é considerada um fenômeno expresso por uma sensação somada a uma reação, essa sensação é medida pelos sistemas nervosos periférico e central e a reação representa a experiência subjetiva que o indivíduo apresenta. É necessário investigar ambos os componentes da definição da dor para compreender a percepção do paciente (Bond, 1986).

Do ponto de vista da MTC, a dor pode ser consequência de uma condição de excesso, causando obstrução da circulação de Qi e de sangue. Exemplos de condições de excesso que contribuem para a dor são as invasões de fatores patogênicos externos, frio ou calor interno, estagnação de Qi ou de sangue, obstrução por fleuma e retenção de alimentos. A 
dor também pode ser causada por condições de deficiência de Qi e sangue e consumo de líquidos corporais em razão de uma deficiência de Yin. Estas condições causam desnutrição dos canais e, então, dor (Klide, \& Gaynor, 2006).

A sensação dolorosa pode ser classificada em aguda e crônica, assim, a dor pode ser percebida de maneiras diferentes pelos sujeitos, uma vez que experiências sensoriais e emocionais desagradáveis podem ser relacionadas a estímulos resultantes de lesões reais ou potenciais (Bond, 1986).

Enquanto a dor aguda é primordial para a preservação da integridade do indivíduo, por ser um sintoma que alerta para a ocorrência de lesões no organismo, a dor crônica não tem esse valor biológico, estabelecendo uma importante causa de incapacidade (Siqueira, \& Teixeira, 2001).

Numerosas são as tentativas de abrandar as dores sofridas pelos indivíduos, desde a antiguidade até as mais recentes pesquisas científicas (Ojugas, 1999).

A responsabilidade de proporcionar alívio da dor e conforto, requer avaliação ampla dos aspectos fisiológicos, emocionais, comportamentais e ambientais que a estimulam ou a exacerbam (Bottega et al., 2014).

A procura por terapias alternativas e complementares tem aumentado progressivamente mais, devido possuir uma comprovada eficácia em diversas patologias, principalmente as que possuem sintomatologia dolorosa, onde o emprego da auriculoterapia pode ser um importante instrumento para a promoção, prevenção e tratamento para saúde, por ser uma técnica de rápida aplicação e fácil adaptação dos pacientes (Correia et al., 2017). Assim, faz-se necessário saber como a terapia auricular é empregada em processos dolorosos?

Como a terapia visa a estimulação de pontos em determinadas regiões da orelha, onde cada uma delas corresponde a áreas do corpo e outras com efeitos mais específicos, e também pelo fato da mesma possuir o efeito reflexo no corpo, a associação destes em protocolos direcionando-os as causas e aos sintomas apresentados pelos pacientes a torna um tratamento alternativo não farmacológica para algias.

Por meio do mecanismo de ação da auriculoterapia, a aplicabilidade de um estímulo no pavilhão auricular pode provocar, por meio de reflexos, reações imediatas ou demoradas, temporárias ou permanentes, passageiras ou definitivas, todas com ação terapêutica (Souza, 2012).

\section{Objetivo Geral}

Realizar uma revisão literária onde possa ser identificado como auriculoterapia é utilizada como processo terapêutico de analgesia em casos de dor.

\subsection{Objetivos Específicos}

- Constatar a eficácia do tratamento com auriculoterapia nas algias;

- Definir quais os pontos do pavilhão auricular são mais utilizados;

- $\quad$ Citar os meios usados para estimulação dos pontos.

\section{Metodologia}

Este trabalho tratou-se de uma pesquisa bibliográfica descritiva, onde foi feita uma análise dos artigos científicos que foram de relevância para este estudo, e aos quais estiveram enquadrados nos critérios de classificação para que pudesse ser realizada a pesquisa.

De acordo com Salomon (2004), a pesquisa bibliográfica é fundamentada em conhecimentos concedidos através da biblioteconomia e documentação, entre outras ciências e técnicas empregadas de forma metódica envolvendo o 
reconhecimento, local e obtenção da informação, fichamento e composição do trabalho científico. Esse processo necessita de uma busca planejada de informações bibliográficas, para assim elaborar e documentar um trabalho de cunho científico.

A busca bibliográfica ocorreu entre os meses de janeiro e fevereiro de 2021. Onde foram utilizadas as plataformas digitais Google Scholar (Google Acadêmico) e biblioteca on-line Scientific Electronic Library Online (SciELO), para ser realizado a busca de artigos relevantes para esta pesquisa. Por meio das palavras chaves: Auriculoterapia / Analgesia / Dor.

Os critérios de inclusão adotados para a busca de artigos foram: artigos que utilizassem a terapia auricular como método terapêutico para tratamento de dores no geral, publicados em português, e que estejam disponíveis em plataformas digitais de forma gratuita. Como critérios de exclusão artigos que abordassem a terapêutica relacionada a dor do trabalho de parto, que estivessem em idioma estrangeiro, e que forem artigos de revisão.

Encontrados 66 artigos sobre a temática na busca inicial, porém ao serem submetidos aos critérios de inclusão e exclusão restaram 15 artigos, após uma leitura minuciosa dos títulos sobraram 7 arquivos para amostra final e realização desta pesquisa de revisão.

Com a leitura dos artigos, foi realizado uma coleta de dados de forma sistematizada e colocados em forma de quadro. Onde de cada material foi retirado os seguintes itens: nome do autor, ano da publicação, título / tema, objetivos, metodologia, pontos usados no tratamento, meio de estimulação usado em cada caso, o número de sessões realizadas para que pudesse ser comprovado a eficácia, e os resultados encontrados.

\section{Resultados e Discussão}

Para demonstrar a abrangência em tratamento de dores, através da auriculoterapia, pode-se observar nos quadros abaixo, os achados dos referidos artigos que foram selecionados conforme os critérios já descritos anteriormente. Abaixo no Quadro 1, serão descritos os estudos realizados para chegar aos resultados e conseguintemente estabelecer discussões.

Quadro 1. Descrição dos artigos onde foi utilizado a auriculoterapia como tratamento para dores.

\begin{tabular}{|c|c|c|c|c|}
\hline AUTOR / ANO & TEMA & OBJETIVO & METODOLOGIA & RESULTADOS \\
\hline Zanelatto, 2013. & $\begin{array}{l}\text { Avaliação da acupressão } \\
\text { auricular na Síndrome do } \\
\text { Ombro Doloroso: estudo } \\
\text { de caso. }\end{array}$ & $\begin{array}{l}\text { Avaliar os resultados da } \\
\text { acupressão auricular } \\
\text { quando usada como terapia } \\
\text { no tratamento da Dor } \\
\text { crônica, secundária à } \\
\text { Síndrome do Ombro } \\
\text { Doloroso, quanto ao efeito } \\
\text { analgésico e terapêutico } \\
\text { satisfatório e o tempo } \\
\text { estimado de tratamento } \\
\text { para obtenção desses } \\
\text { resultados. }\end{array}$ & $\begin{array}{l}\text { Estudo de caso, com } \\
\text { análise quantitativa e } \\
\text { qualitativa. }\end{array}$ & $\begin{array}{l}\text { O estudo demonstrou uma } \\
\text { melhora satisfatória. }\end{array}$ \\
\hline $\begin{array}{l}\text { Ruela et al., } \\
2018 .\end{array}$ & $\begin{array}{l}\text { Efetividade } \\
\text { auriculoterapia na dor de } \\
\text { pessoas portadoras de } \\
\text { câncer em tratamento } \\
\text { quimioterápico: um } \\
\text { ensaio } \\
\text { randomizado. clínico }\end{array}$ & $\begin{array}{l}\text { Avaliar a efetividade da } \\
\text { auriculoterapia na dor de } \\
\text { pacientes com câncer em } \\
\text { tratamento quimioterápico. }\end{array}$ & $\begin{array}{l}\text { Ensaio clínico, } \\
\text { randomizado, } \\
\text { experimental com uso de } \\
\text { questionários. }\end{array}$ & $\begin{array}{l}\text { Observou-se que a } \\
\text { auriculoterapia foi uma } \\
\text { técnica efetiva na redução } \\
\text { da dor oncológica. }\end{array}$ \\
\hline $\begin{array}{l}\text { Ferreira et al., } \\
2018 .\end{array}$ & $\begin{array}{lr}\text { Efetividade } & \text { da } \\
\text { aurículoterapia na dor e } \\
\text { funcionalidade } r \text { de } \\
\text { mulheres com dor } \\
\text { pélvica crônica. }\end{array}$ & $\begin{array}{l}\text { Investigar a efetividade da } \\
\text { auriculoterapia, como } \\
\text { tratamento complementar, } \\
\text { para redução da dor e } \\
\text { melhora funcional em } \\
\text { mulheres com dor pélvica }\end{array}$ & $\begin{array}{l}\text { Ensaio clinico, por meio } \\
\text { experimental e com uso } \\
\text { de questionários. }\end{array}$ & $\begin{array}{l}\text { As avaliações de dor } \\
\text { pélvica, dismenorreia e } \\
\text { funcionalidade } \\
\text { apresentaram melhora } \\
\text { significativa. }\end{array}$ \\
\hline
\end{tabular}




\begin{tabular}{|c|c|c|c|c|}
\hline & & crônica. & & \\
\hline $\begin{array}{l}\text { Bettini; Parisotto, } \\
2018 \text {. }\end{array}$ & 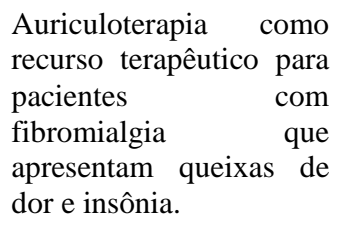 & $\begin{array}{l}\text { Verificar se após } 4 \\
\text { aplicações da técnica de } \\
\text { auriculoterapia, } \\
\text { voluntárias apresentariam } \\
\text { melhoras nos quadros de } \\
\text { dor e insônia. }\end{array}$ & $\begin{array}{l}\text { Pesquisa de campo do } \\
\text { tipo } \\
\text { descritiva, fazendo uso de } \\
\text { questionários para análise. }\end{array}$ & $\begin{array}{l}\text { Apresentou melhora em } \\
\text { ambos os quesitos } \\
\text { observados na pesquisa. }\end{array}$ \\
\hline Silva, 2019. & $\begin{array}{lr}\text { Efeitos } & \text { da } \\
\text { auriculoterapia na } & \text { dor } \\
\text { lombar crônica } & \text { de } \\
\text { profissionais } & \text { de } \\
\text { enfermagem } & \text { em } \\
\text { hospitais } & \text { terciários: } \\
\text { ensaio } & \text { clínico } \\
\text { randomizado } & \end{array}$ & $\begin{array}{l}\text { Avaliar a eficácia da } \\
\text { auriculoterapia com } \\
\text { sementes de mostarda no } \\
\text { tratamento da dor, na } \\
\text { funcionalidade e na } \\
\text { mobilidade lombar de } \\
\text { profissionais técnicos e } \\
\text { auxiliares de enfermagem } \\
\text { com dor lombar crônica. }\end{array}$ & $\begin{array}{l}\text { Um ensaio clínico } \\
\text { randomizado cego, de } \\
\text { forma experimental } \\
\text { através de termogramas } \\
\text { infravermelhos e } \\
\text { algometria por pressão. }\end{array}$ & $\begin{array}{l}\text { Reduziu a assimetria e } \\
\text { temperatura média nos } \\
\text { termogramas analisados e } \\
\text { também aumentou o } \\
\text { limiar de dor, o que } \\
\text { demonstra uma melhora } \\
\text { significativa da dor } \\
\text { lombar no grupo } \\
\text { intervenção. }\end{array}$ \\
\hline $\begin{array}{l}\text { Graça et al., } \\
2020 .\end{array}$ & $\begin{array}{l}\text { Uso da auriculoterapia } \\
\text { no controle da } \\
\text { lombalgia, ansiedade e } \\
\text { estresse de profissionais } \\
\text { do sistema penitenciário }\end{array}$ & $\begin{array}{l}\text { Identificar as contribuições } \\
\text { da auriculoterapia para a } \\
\text { promoção da qualidade de } \\
\text { vida de profissionais do } \\
\text { sistema penitenciário }\end{array}$ & $\begin{array}{l}\text { Estudo experimental, com } \\
\text { coleta de dados. }\end{array}$ & $\begin{array}{l}\text { Verificou-se redução mais } \\
\text { acentuada da intensidade } \\
\text { dos sintomas no grupo } \\
\text { intervenção, } \\
\text { especialmente em relação } \\
\text { ao estresse e lombalgia, o } \\
\text { que aponta a efetividade } \\
\text { da auriculoterapia. }\end{array}$ \\
\hline $\begin{array}{l}\text { Sousa et al., } \\
2020 .\end{array}$ & $\begin{array}{l}\text { Efeito da auriculoterapia } \\
\text { na dor e função sexual } \\
\text { de mulheres com } \\
\text { dismenorreia primária. }\end{array}$ & $\begin{array}{l}\text { Analisar os efeitos da } \\
\text { auriculoterapia nos níveis } \\
\text { de dor e na função sexual } \\
\text { de mulheres com } \\
\text { dismenorreia primária. }\end{array}$ & $\begin{array}{lr}\text { Estudo } & \text { clínico } \\
\text { longitudinal, } & \text { prospectivo } \\
\text { com } & \text { abordagem } \\
\text { quantitativa. } & \end{array}$ & $\begin{array}{l}\text { A auriculoterapia } \\
\text { mostrou-se benéfica em } \\
\text { relação à redução do nível } \\
\text { de dor. }\end{array}$ \\
\hline
\end{tabular}

Fonte: Autores (2021).

Kurebayashi et al. (2012) menciona que a auriculoterapia tem se destacado pelo fato de possuir uma ampla aceitabilidade, eficácia, segurança e reconhecimento em diversos distúrbios.

Nota-se, através do exposto nos quadros 1 e 2, que a auriculoterapia foi utilizada como forma terapeuta em vários tipos de patologias que estão associadas a dor. Alguns casos agudos e outros crônicos, e até mesmo onde a auriculoterapia foi utilizado como forma paliativa. Sendo o único recurso terapêutico usado pelos pesquisadores para tratar as dores, sem auxilio e até mesmo como complemento de demais métodos.

Com a implantação do uso da auriculoterapia, é possível um tratamento vinculado a diversos sinais e sintomas de adoecimentos apresentados pela população, mostrando-se como terapia bem sucedida na manutenção da saúde, indo além do tratamento biomédico, pois é visto sua eficácia no tratamento vinculado à dor pela liberação de endorfinas, controle da inflamação vinculado à ação da acetilcolina e o controle do processamento emocional através do sistema límbico (Hohenberger, \& Dallegrave, 2016).

Em todos os estudos houve resultados satisfatórios e comprovatórios da escolha de tratamento com a terapia auricular para a promoção de analgesia nos processos dolorosos.

Percebe-se que as indicações da auriculoterapia para as desordens do corpo são inúmeras. Seus efeitos benéficos têm ficado evidentes no tratamento da ansiedade, das síndromes dolorosas, das inflamações, das enfermidades endocrinometabólicas, urogenitais, funcionais etc. (Iunes et al., 2015; Kurebayashi et al., 2012; Toca - Villegas et al., 2016; Zanelatto, 2013).

Conforme Kurebayashi (2012), o conhecimento do profissional, assim como, a experiência adquirida na realização da técnica, associada com a prática clínica são fatores imprescindíveis para o alcance de resultados positivos. A seguir no Quadro 
2, faz-se a descrição dos pontos e métodos utilizados em cada artigo estudado, assim como o número de sessões realizadas para efetuação da terapêutica.

Quadro 2. Descrição dos pontos, métodos de estimulação dos pontos e o número de sessões efetuadas.

\begin{tabular}{|c|c|c|c|c|c|}
\hline AUTOR / ANO & TEMA & $\begin{array}{c}\text { TIPO / LOCAL DA } \\
\text { DOR }\end{array}$ & $\begin{array}{l}\text { PONTOS } \\
\text { USADOS }\end{array}$ & $\begin{array}{c}\text { MÉTODO DE } \\
\text { ESTIMULAÇÃO }\end{array}$ & $\begin{array}{l}\text { NÚMERO DE } \\
\text { SESSÕES }\end{array}$ \\
\hline Zanelato, 2013. & \begin{tabular}{lr} 
Avaliação & da \\
acupressão & \\
auricular & na \\
Síndrome & do \\
Ombro Doloroso: \\
\multicolumn{2}{l}{ estudo de caso. }
\end{tabular} & $\begin{array}{l}\text { Síndrome do ombro } \\
\text { doloroso; }\end{array}$ & $\begin{array}{l}\text { Shen men; Rim; } \\
\text { Simpático; Ombro; } \\
\text { Articulação do } \\
\text { ombro; Clavícula. }\end{array}$ & Esferas de cristais. & $\begin{array}{l}\text { Doze (12) sessões, } 1 \mathrm{x} \\
\text { por semana. }\end{array}$ \\
\hline $\begin{array}{l}\text { Ruela et al., } \\
2018 .\end{array}$ & $\begin{array}{lr}\text { Efetividade } & \text { da } \\
\text { auriculoterapia } & \text { na } \\
\text { dor de pessoas } \\
\text { portadoras } & \text { de } \\
\text { câncer } & \text { em } \\
\text { tratamento } & \\
\text { quimioterápico: } & \text { um } \\
\text { ensaio clínico } \\
\text { randomizado. }\end{array}$ & Dor oncológica. & $\begin{array}{l}\text { Shen men; Rim; } \\
\text { Simpático. }\end{array}$ & $\begin{array}{l}\text { Agulha } \\
\text { semipermanente. }\end{array}$ & $\begin{array}{l}\text { Oito }(08) \text { sessões, } 1 \mathrm{x} \\
\text { por semana }\end{array}$ \\
\hline $\begin{array}{l}\text { Ferreira et al., } \\
2018 .\end{array}$ & $\begin{array}{lr}\text { Efetividade } & \text { da } \\
\text { aurículoterapia } & \text { na } \\
\text { dor } & \mathrm{e} \\
\text { funcionalidade } & \text { de } \\
\text { mulheres com dor } & \text { dólvica crônica. }\end{array}$ & Dor pélvica. & $\begin{array}{l}\text { Shen man; Rim; } \\
\text { Fígado; } \\
\text { Útero. }\end{array}$ & $\begin{array}{l}\text { Sementes } \\
\text { mostarda. }\end{array}$ & $\begin{array}{l}\text { Cinco } \\
\text { aplicações, sendo } 1 \mathrm{x} \\
\text { na semana. }\end{array}$ \\
\hline $\begin{array}{l}\text { Bettini; } \quad \& \\
\text { Parisotto, 2018. }\end{array}$ & $\begin{array}{l}\text { Auriculoterapia } \\
\text { como recurso } \\
\text { terapêutico para } \\
\text { Pacientes com } \\
\text { fibromialgia que } \\
\text { apresentam } \\
\begin{array}{l}\text { Queixas de dor e } \\
\text { insônia }\end{array}\end{array}$ & Fibromialgia. & $\begin{array}{l}\text { Shen men; Rim; } \\
\text { Simpático; } \\
\text { Suprarrenal. }\end{array}$ & $\begin{array}{l}\text { Sementes de } \\
\text { mostarda e agulhas } \\
\text { semipermanente. }\end{array}$ & $\begin{array}{l}\text { Quatro (04) sessões, } \\
\text { 1x por semana. }\end{array}$ \\
\hline Silva, 2019. & $\begin{array}{l}\text { Efeitos da } \\
\text { auriculoterapia na } \\
\text { dor lombar crônica } \\
\text { de profissionais de } \\
\text { enfermagem em } \\
\text { hospitais terciários: } \\
\text { ensaio clínico } \\
\text { randomizado }\end{array}$ & Dor lombar. & $\begin{array}{l}\text { Shen men; Rim; } \\
\text { Simpático; Lombar } \\
\text { (coluna). }\end{array}$ & $\begin{array}{l}\text { Sementes } \\
\text { mostarda. }\end{array}$ & $\begin{array}{l}\text { Quatro (04) sessões, } \\
\text { 1x na semana. }\end{array}$ \\
\hline $\begin{array}{l}\text { Graça et al., } \\
2020 .\end{array}$ & \begin{tabular}{ll} 
Uso & da \\
auriculoterapia & no \\
controle & da \\
lombalgia, & \\
ansiedade e estresse \\
\multicolumn{2}{l}{ de profissionais do } \\
sistema & \\
penitenciário. & \\
\end{tabular} & Dor lombar. & $\begin{array}{l}\text { Shen men; Rim; } \\
\text { Diafragma; Fígado; } \\
\text { Coração; Tronco } \\
\text { cerebral; Ansiedade. }\end{array}$ & $\begin{array}{l}\text { Sementes } \\
\text { mostarda. }\end{array}$ & $\begin{array}{l}\text { Seis }(06) \text { sessões, } 1 \mathrm{x} \\
\text { por semana. }\end{array}$ \\
\hline $\begin{array}{l}\text { Sousa et al., } \\
2020 .\end{array}$ & $\begin{array}{ll}\text { Efeito } & \text { da } \\
\text { auriculoterapia na } \\
\text { dor e função sexual } \\
\text { de mulheres com } \\
\text { dismenorreia } \\
\text { primária. }\end{array}$ & $\begin{array}{l}\text { Cólica menstrual } \\
\text { (dismenorreia } \\
\text { primaria). }\end{array}$ & $\begin{array}{l}\text { Shen men; Rim; } \\
\text { Simpático; Ovário; } \\
\text { Útero; Endócrino; } \\
\text { Fígado (em alguns } \\
\text { casos, substituindo } \\
\text { o simpático). }\end{array}$ & $\begin{array}{l}\text { Agulha } 0,25 / 15 \mathrm{~mm} \text {, } \\
\text { e micropore. }\end{array}$ & $\begin{array}{lcc}\text { Vinte e } & \text { duas } & (22) \\
\text { sessões, } & 2 \mathrm{x} & \text { por } \\
\text { semana. } & & \end{array}$ \\
\hline
\end{tabular}


Percebe-se que no Quadro 2, os autores em seus protocolos, a maioria fez uso de sementes de mostarda, apenas alguns usaram agulhas, esferas e micropore para simular a estimulação nos pontos auriculares. A preferência pelo meio de estimulo varia de acordo com as características do tipo de dor, como nos casos em que ela se apresenta com mais intensidade e causa mais incomodo aos participantes.

A terapia é realizada através da estimulação dos pontos, sendo capaz de abranger a aplicação da pressão digital, sementes de vaccaria ou mostarda torrada, agulhas, esferas, discos magnéticos, estimulação elétrica ou laser (Round, Litscher, \& Bahr, 2013).

Já em relação aos pontos usados, cada protocolo mostrou-se eficaz em relação aos tipos de disfunções que foram empregados. Havendo prevalência de alguns pontos em todos os estudos, como na utilização do Shen men/ Rim/ Simpático em praticamente todos os casos.

O ponto Shen men age na estimulação do tronco e córtex cerebral, na qual atua no processo de receber, decodificar, modular e condicionar os reflexos dos próximos pontos estimulados, impedindo desequilíbrios e novas patologias, agindo também através da analgesia proporcionada por meio da liberação de endorfinas, sendo um dos principais pontos a ser utilizado no tratamento de alivio da dor (Ruela et al., 2018).

Segundo Souza (2012), o ponto do Rim, entende-se que sua ação analgésica pode estar relacionada a ativação de retiradas de toxinas, redução de linfedemas e ao aumento da capacidade de oxigenação dos tecidos.

Em relação ao ponto simpático, atua na promoção do equilíbrio geral do organismo, acelerando e regulando a atividade neurovegetativa, equilibrando as funções simpáticas e parassimpáticas do indivíduo ao promover o reequilíbrio do sistema nervoso autônomo, mostrando-se de alta importância na promoção da analgesia, relaxamento de fibras musculares e ação anti-inflamatória (Ruela et al., 2018).

Os demais pontos foram diferenciados pelo emprego da região ou relação com o órgão alvo, e até mesmo sua correlação/ simbolismo na MTC, para seu uso conforme a interpretação com base nos preceitos dos 5 elementos.

O diagnóstico auricular é realizado através da inspeção e palpação, buscando por alterações na anatomia auricular e pontos dolorosos que possam estar relacionados com as queixas de saúde do paciente e deverão ser usados no tratamento terapêutico (Hsing, \& Lichun, 2013; Oleson, Kroening, \& Bresler, 1980).

Wang (2005) aponta que o esquema de tratamento e a seleção de pontos baseiam-se no diagnóstico diferencial e destaca que uma mesma patologia pode ter tratamentos diferentes, assim como patologias diferentes podem ter o mesmo tratamento.

Para que conseguisse chegar aos resultados almejados, os pesquisadores protocolaram o número de sessões em que atingiram o efeito desejado pela auriculoterapia, a depender da patologia. Em média foram atendimentos semanais em que se passava no período mínimo de um mês.

Existem evidências que a auriculoterapia é uma modalidade promissora na redução imediata da dor (após 48 horas da primeira aplicação) e sem efeitos colaterais importantes (Murakami, Fox, \& Dijkers, 2017).

Em um dos casos analisados, pode-se notar que com o alivio da dor consequentemente houve influência para a regressão de outro quadro clinico apresentado pelos participantes.

Moura et al. (2017), afirma que, a avaliação abrangente da dor é essencial para identificar, além da intensidade, os fatores sociais, biológicos, culturais e psicológicos que a influenciam, bem como os impactos negativos, na qual essa experiência pode causar na vida dos acometidos.

Tal qual, Sator-Katzenschlager et al. (2004), diz que a acupuntura auricular pode alterar outros fenômenos relacionados à dor crônica, como o bem-estar psíquico, o nível de atividade, a qualidade do sono, a quantidade de medicamentos ingeridos e até mesmo facilitar o retorno às atividades laborais. 
A MTC possui uma visão holística do ser humano, sendo assim, há uma interação entre todos os órgãos e estruturas e com o lado emocional do indivíduo. Assim, o paciente deve ser tratado como um todo para que ocorra a melhora de um sintoma localizado (Zotelli et al., 2010).

Por ser de fácil manipulação, baixo custo, utilizada em várias disfunções e não possuir efeitos colaterais significativos, a auriculoterapia se mostra ser essencial para a população, já que a mesma para ser realizada dispões de diversos métodos de estimulação tornando-a acessível. Servindo tanto para a promoção, prevenção, tratamento e auxílio na reabilitação.

A utilização das práticas integrativas e complementares, são de grande importância no processo de reabilitação e construção da saúde do indivíduo, isso é necessário por identificação de um ser não somente visto como um agregado de matéria, mas que tenha sentimentos e pensamentos, que são estruturas energéticas e não químicas, atentando o profissional aos adoecimentos de origem energética, podendo assim, expor o indivíduo ao tratamento adequado em relação à origem de sua enfermidade apresentada (Galli et al., 2012).

\section{Conclusão}

O tratamento da dor é de extrema importância, principalmente para o bem estar geral, como para o equilíbrio do corpo e para a manutenção das atividades diárias, já que este processo afeta todos os campos da vida do ser humano.

O mecanismo da MTC que observa o corpo de forma holística, não busca a cura só pelo tratamento de uma característica e sim pelo conjunto de fatores que a causaram, pode ser observado sua efetividade através do uso da auriculoterapia, onde demonstrou que utilizando esse microssistema conseguiu-se amenizar e reverter o processo doloroso nos casos estudados.

Portanto, a auriculoterapia, além de ser considerada uma técnica, na qual proporciona um tratamento rápido e simples, também poderá ser utilizada como um método terapêutico bastante proveitoso e eficaz para algias. Entretanto, necessita-se de métodos organizados para o desenvolvimento de estudos, e assim concretizar os indícios encontrados em grande parte das evidências cientificas.

\section{Referências}

Bettini, S. M., \& Parisotto, D. (2018). Auriculoterapia como recurso terapêutico para pacientes com fibromialgia que apresentam queixas de dor e insônia. Revista UNIANDRADE, 12(1), 21-27.

Boleta - Ceranto, D. C. F. et al. (2008). O efeito da acupuntura no controle da dor na odontologia. Arq. Ciênc. Saúde Unipar, Umuarama, $12(2)$, 143-148.

Bond, R. M. (1986). Dor: natureza, análise e tratamento. Rio de Janeiro (RJ): Colina Editora.

Bottega, F. H. et al. (2014). Avaliação da dor em neonatos e crianças em terapia intensiva. Revista de Pesquisa: Cuidado e Fundamental, 6(3), 909-9017.

Brisola, A. M. et al. (2016). Acupuntura e auriculoterapia como métodos não farmacológicos de alívio da dor no processo de parturição. Cogitare Enferm, 21(3), 1-2.

Corrêa, P. M. T. et al. (2017). Auriculoterapia como intervenção para redução da dor em estudantes da unipampa campus uruguaiana. Anais do $9^{\circ}$ Salão Internacional de Ensino, Pesquisa e Extensão - SIEPE Universidade Federal do Pampa | Santana do Livramento.

Ferreira, D. A. et al. (2018). Efetividade da aurículoterapia na dor e funcionalidade de mulheres com dor pélvica crônica. Trabalho de Conclusão de Curso (TCC) em fisioterapia. Atlas.

Galli, K. S. B. et al. (2012). Saúde e equilíbrio através das terapias alternativas: Relato de experiência. Revista de Enfermagem, 8(8), $245-255$.

Gori, L., \& Firenzuoli, F. (2007). Ear Acupuncture in European Traditional Medicine. Evid Based Complement Alternat Med. Periódico, 4(1), 13-16.

Graça, B. C. et al. (2020). Uso da auriculoterapia no controle da lombalgia, ansiedade e estresse de profissionais do sistema penitenciário. BrJP, 3(2), 142-146.

Hohenberger, G. F., \& Dallegrave, D. (2016). Auriculoterapia para profissionais de saúde: Percursos possíveis da aprendizagem à implantação na Unidade de Saúde. Saúde em Redes, 2(4), 372-382.

Hsing, Y. C., \& Lichun, H. (2013). Comprehensive and Systematic Auricular Diagnosis Protocol. Medical Acupuncture, 25(6), $423-436$. 
Research, Society and Development, v. 10, n. 12, e400101220517, 2021

(CC BY 4.0) | ISSN 2525-3409 | DOI: http://dx.doi.org/10.33448/rsd-v10i12.20517

Iunes, D. H. et al. (2015). Role of Auriculotherapy in the treatment of temporomandibular disorders with anxiety in university students. Evidence-Based Complementary and Alternative Medicine, 2015, 1-9.

Klide, A. M., \& Gaynor, J. S. (2006). Acupuntura para analgesia cirúrgica e pós-operatória. SHOEN, AM Acupuntura veterinária. Da arte antiga à medicina moderna, 2, 289-295.

Kurebayashi, L. F. S. (2012). Eficácia da auriculoterapia para estresse segundo experiência do terapeuta: ensaio clínico. Acta Paulista de Enfermagem, 25(5), 694-700.

Kurebayashi, L. F. S. et al. (2012). Aplicabilidade da auriculoterapia com agulhas ou sementes para diminuição de estresse em profissionais de enfermagem. Revista da Escola de Enfermagem da USP, 46(1), 89-95.

Ma, Y., Ma, M., \& Cho, Z. H. (2006). Acupuntura para controle da dor: um enfoque integrado. Editora Roca, 1, 31-47. Atlas.

Meirelles, M. P. M. R., Gonçalo, C. S., \& Sousa, M. L. R. (2009). Manejo da dor orofacial através do tratamento com acupuntura: relato de um caso. Rev Odontol UNESP, 38(6), 379-382.

Moura, C. C. et al. (2017). Impactos da dor crônica na vida das pessoas e a assistência de enfermagem no processo. Avances en Enfermería, 35(1), 53-62.

Murakami, M., Fox, L., \& Dijkers, M. P. (2017). Ear Acupuncture for Immediate Pain Relief-A Systematic Review and Meta-Analysis of Randomized Controlled Trials. Pain Med., 18(3), 551-564.

Ojugas, A. C. (1999). A dor através da história e da arte. Cleveland: Atlas Medical Publishing, 159.

Oleson, T. D., Kroening, R. J., \& Bresler, D. E. (1980). An experimental evaluation of auricular diagnosis: the somatotopic mapping or musculoskeletal pain at ear acupuncture points. Pain, 8(2), 217-229.

Round, R., Litscher, G., \& Bahr, F. (2013). Auricular acupuncture with laser. Evid Based Complement Alternat Med., 2013, 1-22.

Ruela, L. O. et al. (2018). . Efetividade da auriculoterapia na dor de pessoas portadoras de câncer em tratamento quimioterápico: um ensaio clínico randomizado. Rev Esc Enferm USP, 52(03402).

Salomon, D. V. (2004). Como fazer uma monografia. (11a ed.),. Atlas.

Sator-Katzenschlager, S. M. et al. (2004). The short- and long-term benefit in chronic low back pain through adjuvant electrical versus manual auricular acupuncture. Anesth Analg, 98(5), 1359-1364.

Silva, A. P. G. (2019). Efeitos da auriculoterapia na dor lombar crônica de profissionais de enfermagem em hospitais terciários: ensaio clínico randomizado. Dissertação de Mestrado. Atlas.

Siqueira, J. T. T., \& Teixeira, M. J. (2001). Dor orofacial: diagnóstico, terapêutica e qualidade de vida. Editora Maio. Atlas.

Sousa, F. F., Sousa Júnior, J.F. M., \& Ventura, P. L. (2020). Efeito da auriculoterapia na dor e função sexual de mulheres com dismenorreia primária. BrJP, $3(2), 127-130$.

Souza, M. P. (2012). Tratado de Auriculoterapia. Look, 358. Atlas.

Toca-Villegas, J. et al. (2016). Eficacia de la auriculoterapia modificada como tratamento para el control del dolor postoperatorio em pacientes intervenidos mediante colecistectomía laparoscópica. Cirugía y Cirujanos, 85(3), 220-224.

Wang, L. G. (2005). Tratado Contemporâneo de Acupuntura e Moxibustão. CEIMEC, 618. Atlas.

Zanelatto, A. P. (2013). Avaliação da acupressão auricular na Síndrome do Ombro Doloroso: estudo de caso. Revista Brasileira de Enfermagem, 66(5), 694701.

Zotelli, V. L. R., Meirelles, M. P. M. R., \& Sousa, M. L. R. (2010). Uso da acupuntura no manejo da dor em pacientes com alterações na articulação temporomandibular (ATM). Revista de Odontologia da Universidade Cidade de São Paulo, 22(2), 185-188. 